تأثير إضافة الشب على كفاءة المعالجة البيولوجية وإزالة الفوسفات من المطروحات

\author{
زينه فخري الهاتثي' ، حنان حقي إسماعيل الخياط'

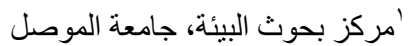

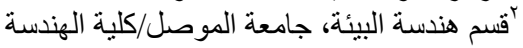

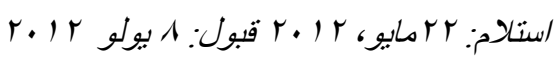

ت تلخلاصة

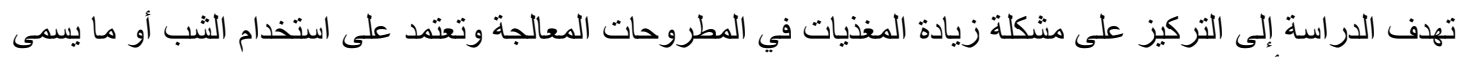

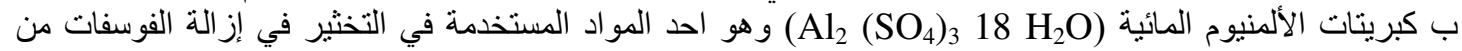

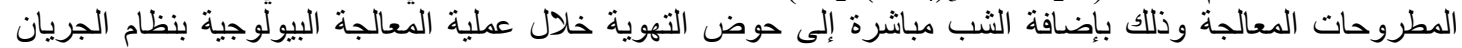

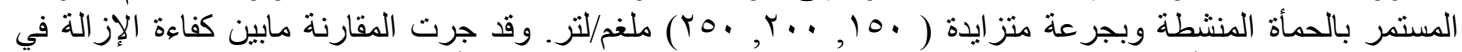

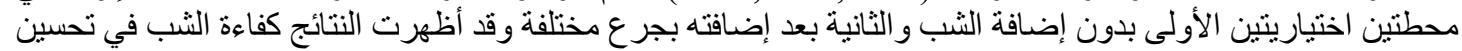

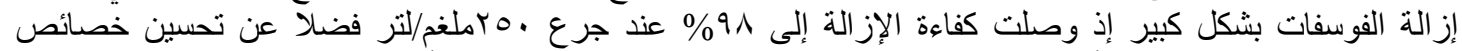

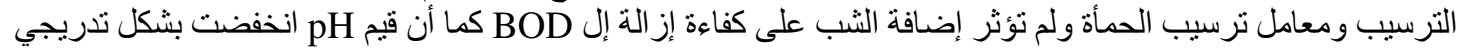

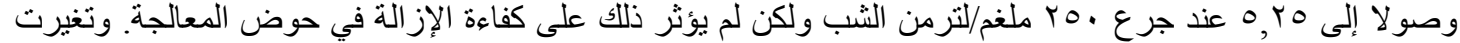

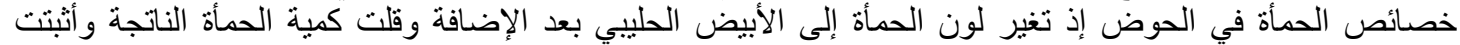

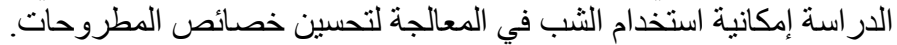
الكلمات المفتاحية: الثب، المغذيات، الحمأة المنشطة، المعالجة البيولوجية، الفوسفات.

حوضي معالجة بيولوجية بإضافة الثب للحوض للحوض الأول فقط تحت نفس ظروف بولئ المعالجة.

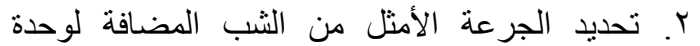

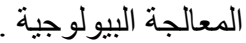

r. در اسة تأثثر إضافة الثبرلبة على معامل ترسيب الحمأة وتحسين خصائص الترسيب عند الجرع (SVI)

المختلفة.

الار اسات السابقة:

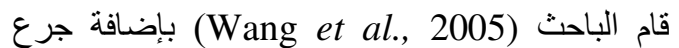

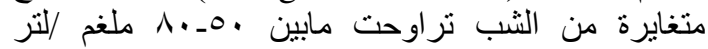

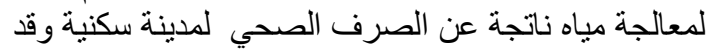

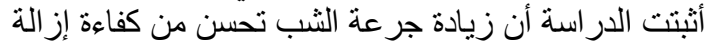

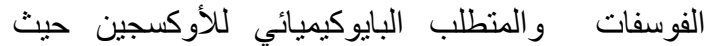

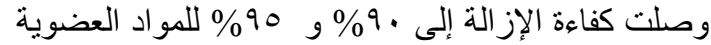

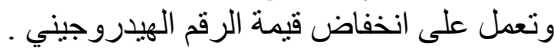

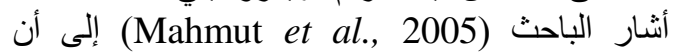

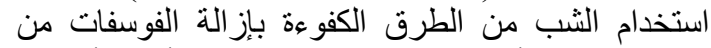

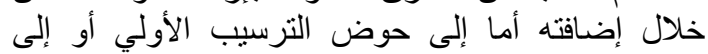

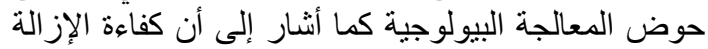

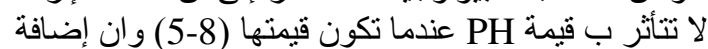
2.3 مول من الثب تعمل على إز الة الثمان مول من الفوسفات.

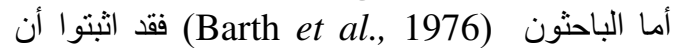

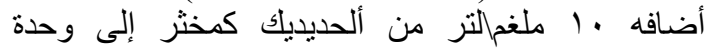

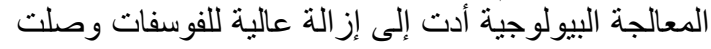

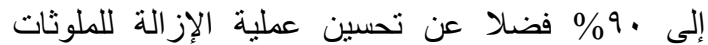

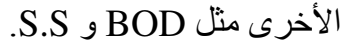

أما الباحثون (Gersbery et al., 1988)

بدراسة حول إضافة الشب إلى وحدة المعالجة البيولوجية

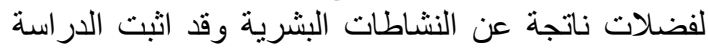

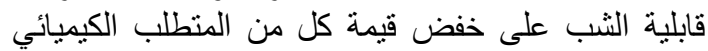
للأوكسجين (COD) للمياه المعالجة حيث كلث كانت (20)
المقدمة:

أدت زيادة نسبة المغذيات الناتجة عن الفضلات المدنية

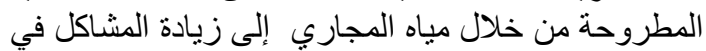

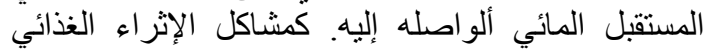

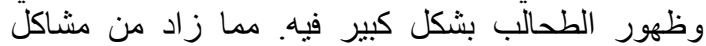

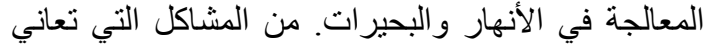

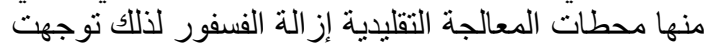
الدراسات إلى إيجاد حلول مختلفة لتقليل تركيز الفئلة الفسفور

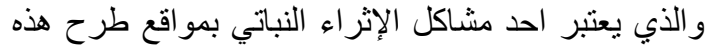
المياه,تناول بحثنا احد الحلول المقترحة لتقليل تركيز

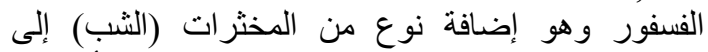

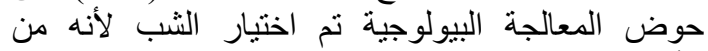

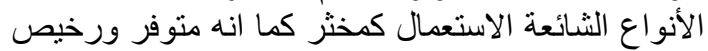

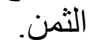

وقد استخدم العديد من الباحثين المخثرات كأحد الحلول

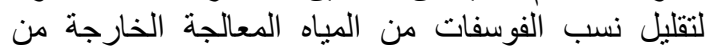

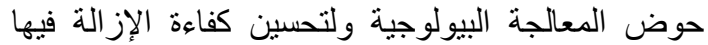

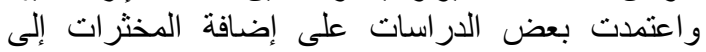

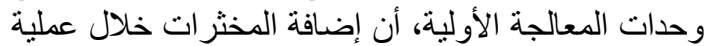

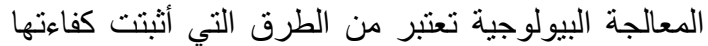

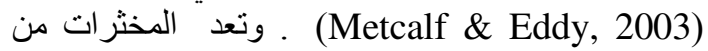

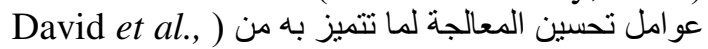

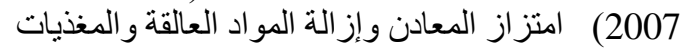

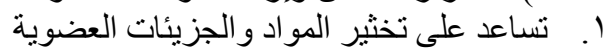

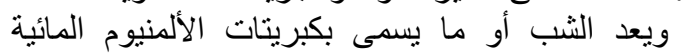
من المخثر ات التي أثتبت كفاءتها $\left(\mathrm{Al}_{2}\left(\mathrm{SO}_{4}\right)_{3} 18 \mathrm{H}_{2} \mathrm{O}\right)$

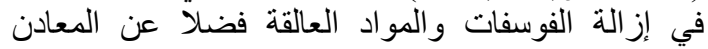
والّي إد العضوية العويفات

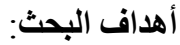

1. دراسة تأثثير إضافة الثب على كفاءة إز الة الملوثات

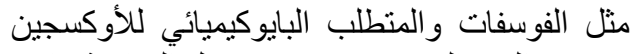
وخفض الرقم الهيدروجيني .من خلال ألمقارنة مابين لفين

\begin{tabular}{ll}
\hline * Corresponding author: & 63 \\
Dr. Hanan Haqe & 63 \\
$\varangle$ hanan_eng2002@yahoo.com &
\end{tabular}


للمحطة الاختيارية تماثيا مع عمل محطات معالجة مياه الفضلات الكبيرة التي تعمل في معظمها بهذا الأسلوب.

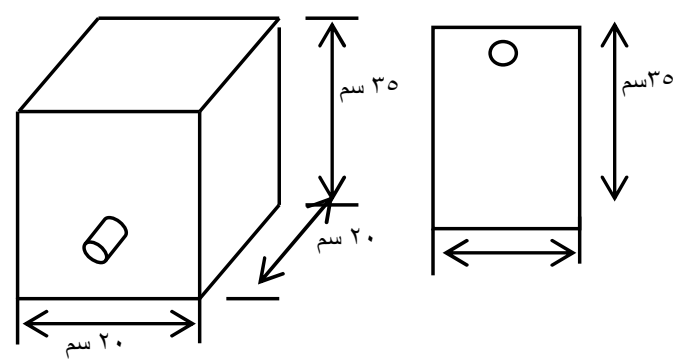

مخطط(1) الحوض الزجاجي المستخدم بالمعالجة البيولوجية.

الفحوصات المختبرية:

أجريت الفحوصات المختبرية بالاعتماد على الطرق القياسية (AWWA; WPCF 1998)

ا. فحص الرقم الهروجيني ( pH ) : بالاعتماد

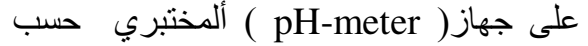
الفقرة (H4500).

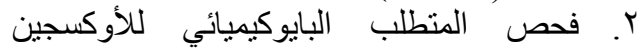

:Biological Oxygen Demand :BOD

تم هذا الفحص بالاعتماد على الفقرة (507).

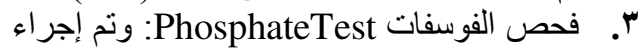

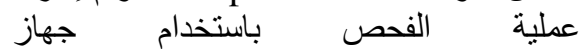

Ultra Violet Spectro Photometer

424 Screening Method

(E)

؛. فحص المواد العالقة S.S وتم العمل حسب

(2540A, 2540D) الفقرة

فحص معامل ترسيب الحماة SVI تم هذاة الفحص

بالاعتماد على ما قام به الباحث (Plaza et al., 1997)

النتائتج والمناقشة:

تم خلال هذا البحث استخدام مياه

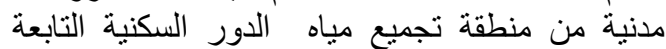

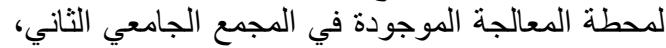

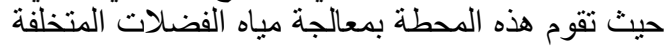
عن الدور السكنية التابعة للمجمع و الكليات المجاورة المدة المبنات

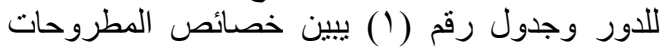

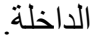

ملغم/لتر أما الفوسفات فقد كانت القيمة اقل من 0.5ملغم

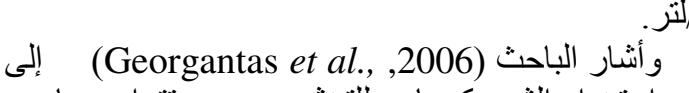

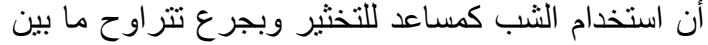

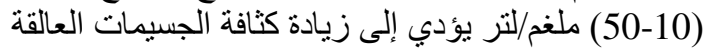

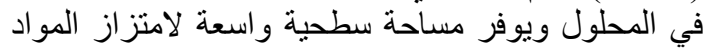
العضوية كما يحسن من إز الة الفوسفات ملهن

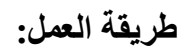

تم اخذ مطروحات لفضلات العلة دور سكنية واجراء الفحوصات المختبرية عليها لمعرفة خصائصسها و إدخالها

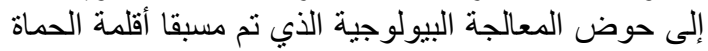

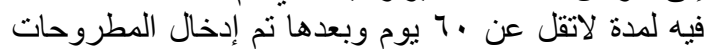

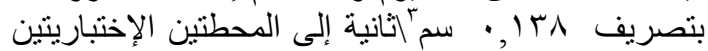

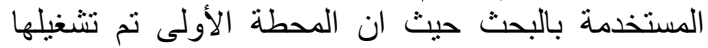

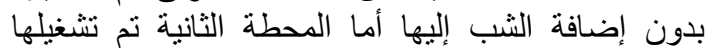

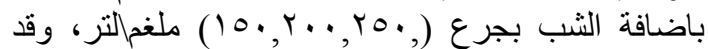

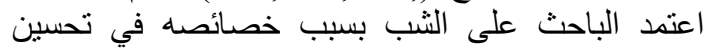

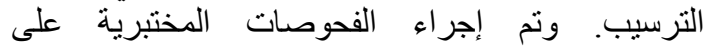

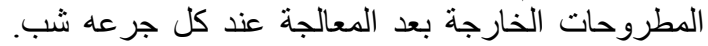

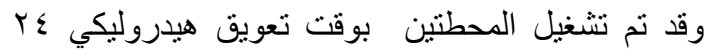

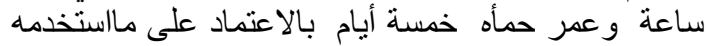

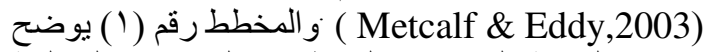
حوض التهوية المستخدم بالمحطتين المستخدم بالمعالجة

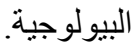
حوض التهوية:

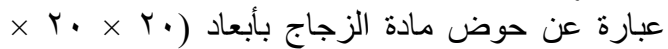

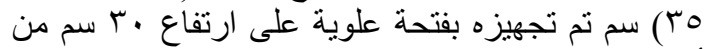

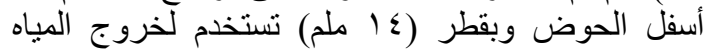

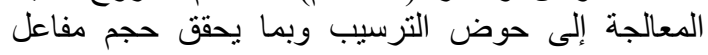

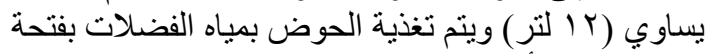

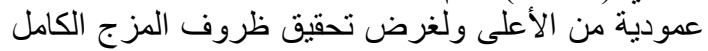

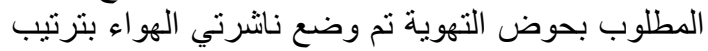

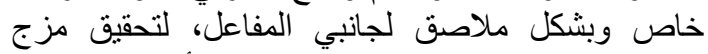
لمكونات الحوض ونوزيع منساو لتركيز الأوكسجين داخل حوض التهوية حوض الترسيب:

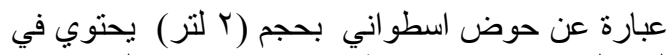

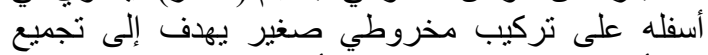
الحمأة المترسبة. تم اعتماد أسلوب التشغيل المستمر

\begin{tabular}{|c|c|c|c|c|c|}
\hline 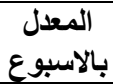 & الأكرار خلال & عدرة النماذج طول & المدى (التركيز الداخل) & الوحدة & الخصائص \\
\hline 1 & 3 & 10 & $425-60$ & $\mathrm{mg} / \mathrm{l}$ & $\begin{array}{c}\text { المتطلب البايوكيميائي } \\
\text { BOD5 }\end{array}$ \\
\hline 1 & 3 & 10 & $320-200$ & $\mathrm{mg} / \mathrm{l}$ & S S المواد العالقة \\
\hline 1 & 3 & 10 & $9.4-2.7$ & $\mathrm{mg} / \mathrm{l}$ & الفوسفات PO4 \\
\hline 1 & 3 & 10 & $7.8-6$ & & الرقم الهيدروجيني PH \\
\hline 1 & 3 & 10 & $15-38$ & $\mathrm{mg} / \mathrm{l}$ & $\mathbf{N H}_{3}$ \\
\hline 1 & 3 & 10 & $640-100$ & $\mathrm{mg} / \mathrm{l}$ & COD \\
\hline
\end{tabular}

جدول ( ) : خصائص المطروحات الداخلة. 


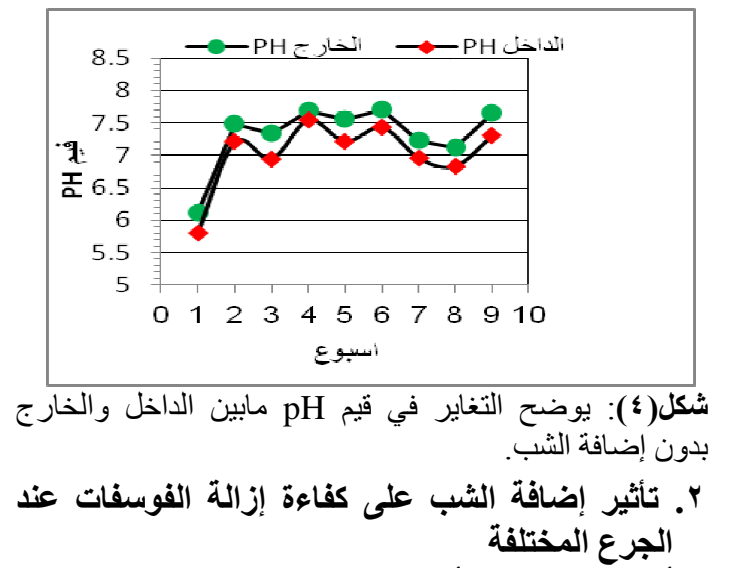

أظهرت الدراسة أن إضافة الثب بشكل عام تزيد من الثناه

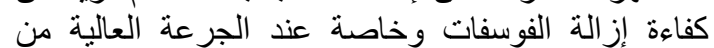

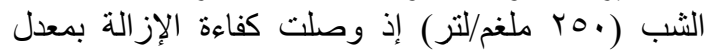

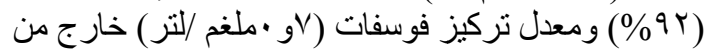

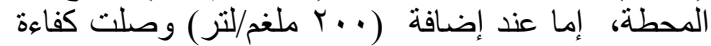

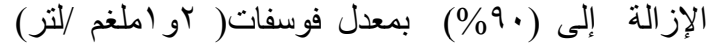

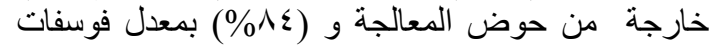

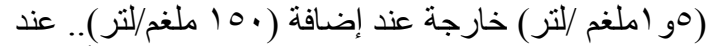

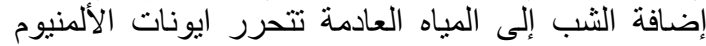

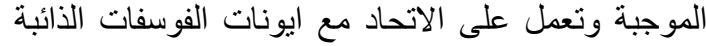
بالماء فتكون مادة عالقة تترسب مع الحماة وحسب الحمات المعادلة

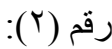

\section{$\mathrm{AL}^{3+}+\mathrm{PO}_{4} \leftrightarrow \mathrm{ALPO}_{4}+\mathrm{H}^{+} \quad$ (2)}

وهذا يتفق مع ما توصل إليه..Lujubinkoet al, 2004), (Juker et al.,2007),( Metcalf \& Eddy 2003). و الأشكال (0_9) توضح ذلك .

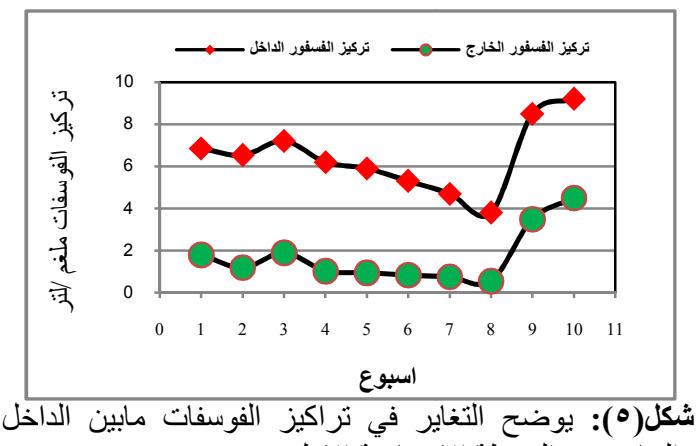
والخارج في المحطة الاختبارية الاولى. تيلى

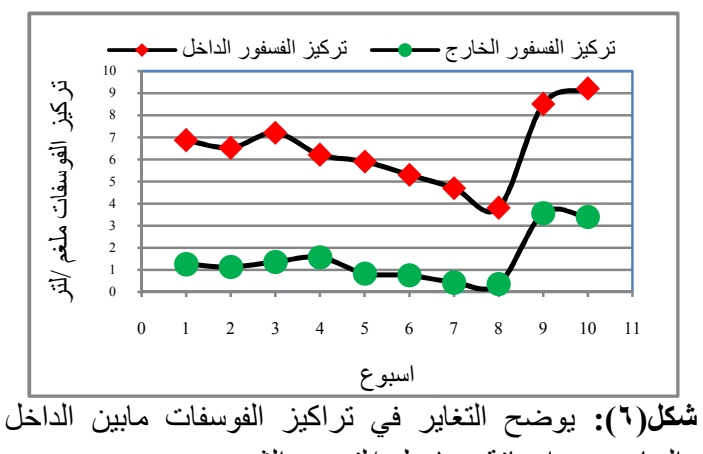

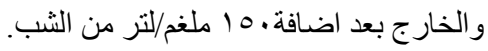

أ تأثير إضافة الثب على قيم الرقم الهيدوجيني pH عند الجرع المختلفة

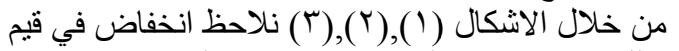

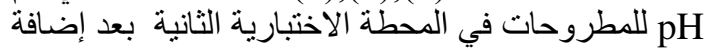

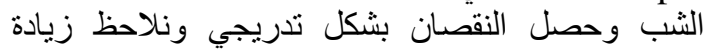

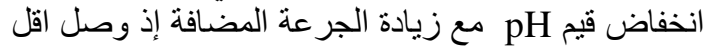

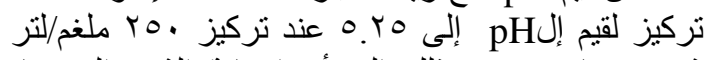
شب مضاف يعزى ذللى إلى أن إضافة الثب تبل إلى مياه

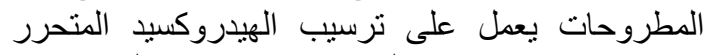

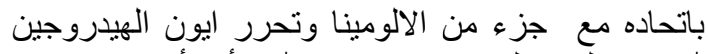

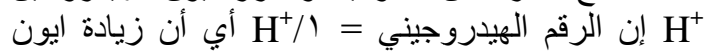

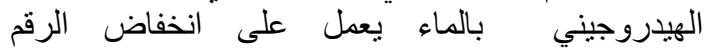

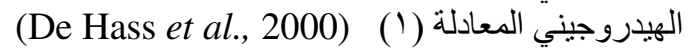

$$
\mathrm{Al}^{+3}+3 \mathrm{H}_{2} \mathrm{O} \rightarrow \mathrm{Al}(\mathrm{OH})_{3}+3 \mathrm{H}^{+} \text {(1) }
$$

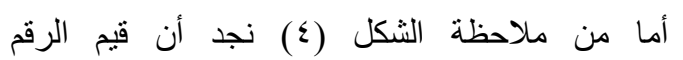

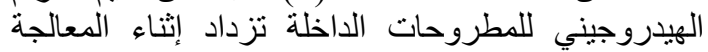

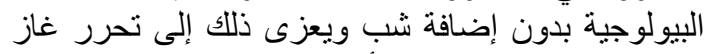
ثاني اوكسيد الكربون نتيجة أكسدة المو واد العضوية.

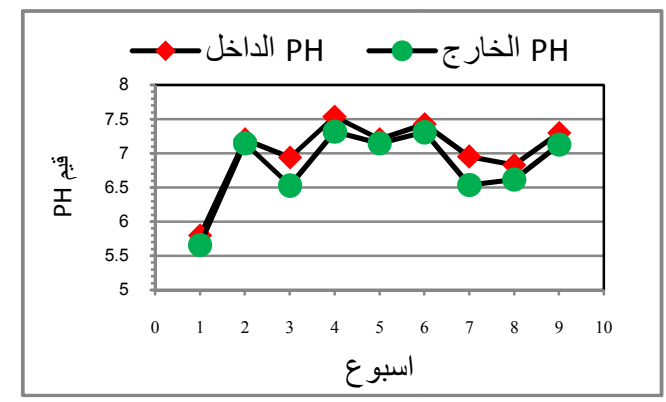

شكل(1) (1): يوضح التغاير في قيم pH مابين الداخل و الخارج بعد إضافة . 10 ملغم/لتر من الثب.

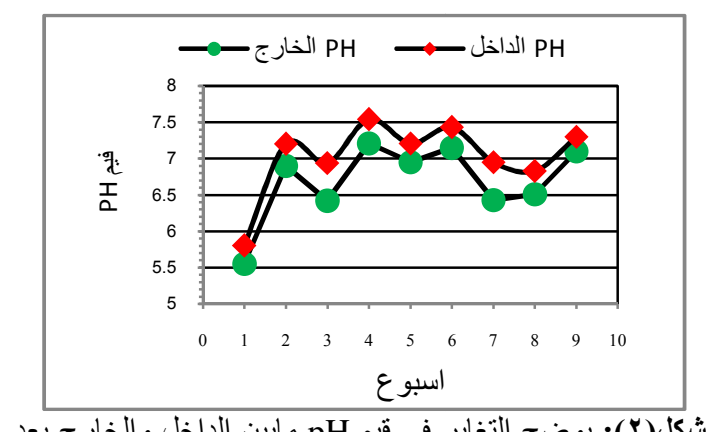

شكل(ץ): يوضح التغاير في قيم pH مابين الداخل و الخارج بعد إضافة . . . ملغم/لتر من الثب.

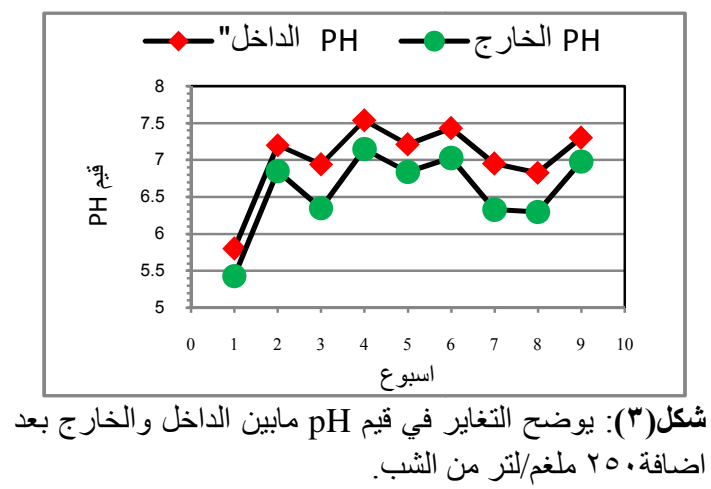




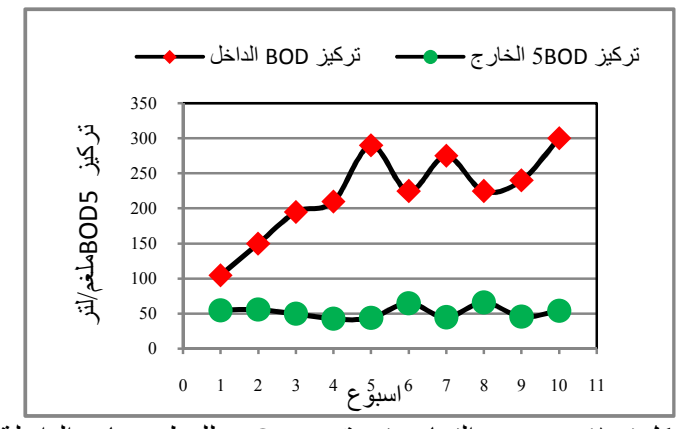

شكل(• (1): يوضح التغاير في قيم BOD و الخارجة من المحطة إلا ختبارية الأولى.

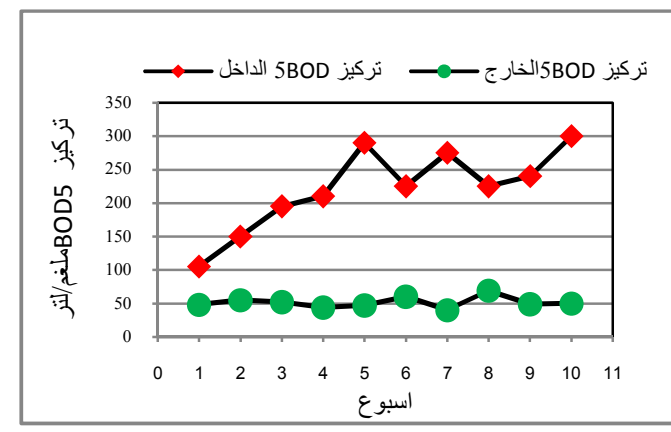

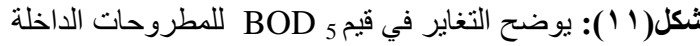

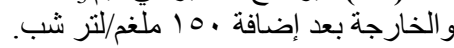
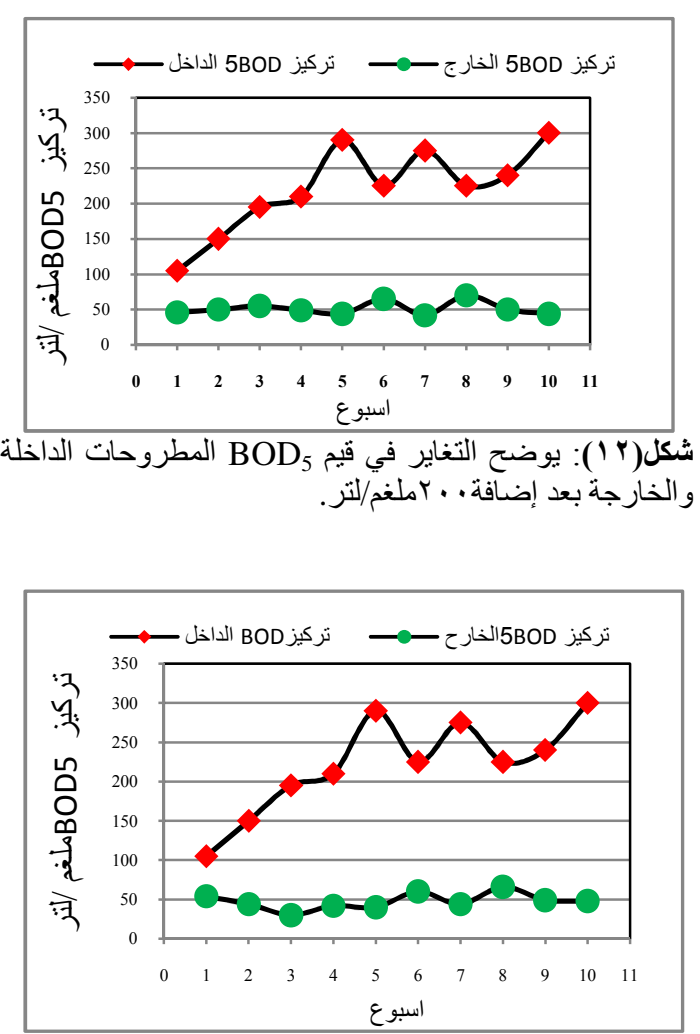

شكل(r I ): يوضح التغاير في قيم 5 BOD المطروحات الداخلة

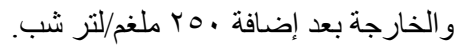

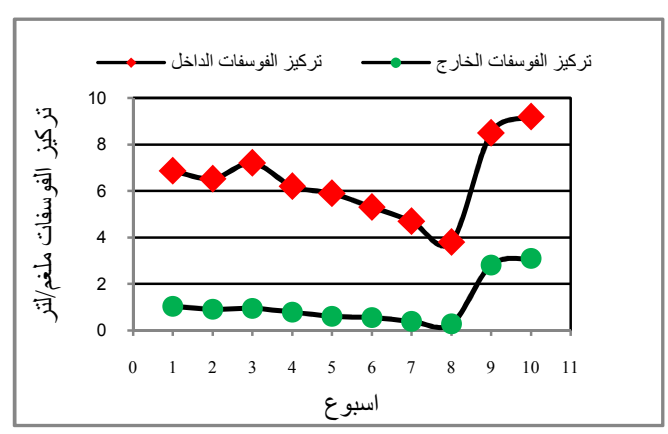

شكل(V): يوضح التغاير في تراكيز الفوسفات مابين الداخل

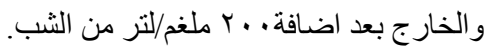

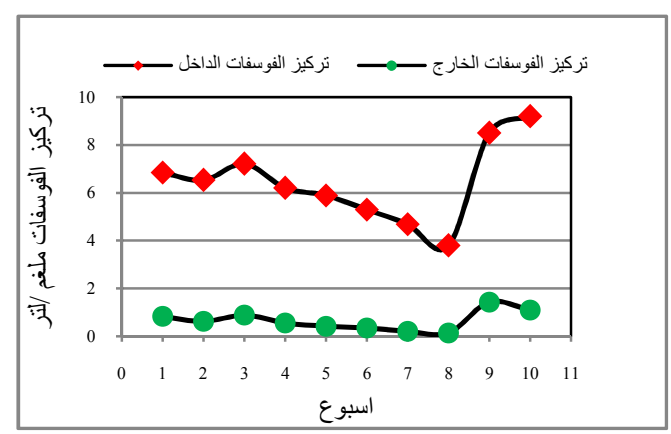

شكل(^): يوضح التغاير في نراكيز الفوسفات مابين الداخل

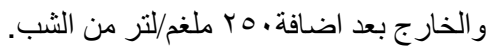

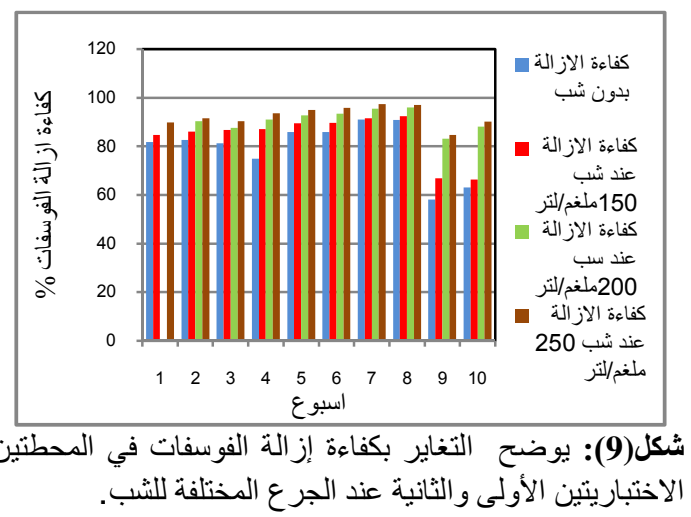

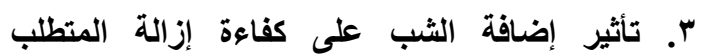
البايوكيميائي للأوكسجين (B0D5) عند الجرة الجرع المختلفة:

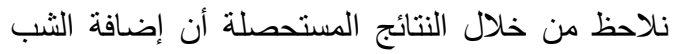

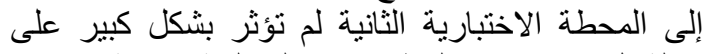

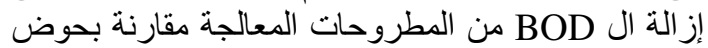

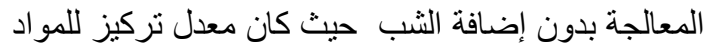

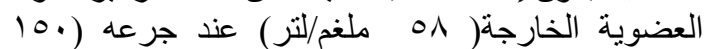

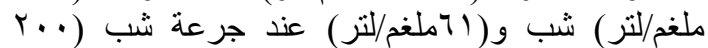

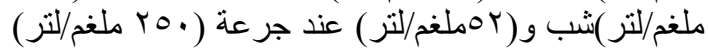

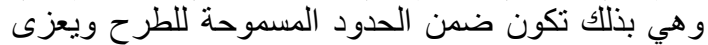

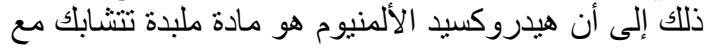

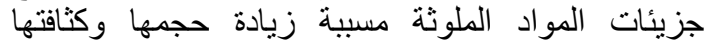

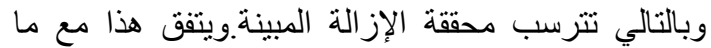

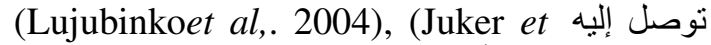

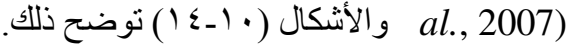




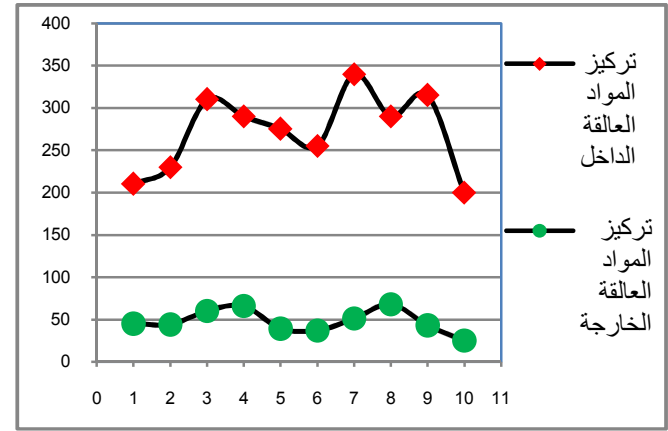

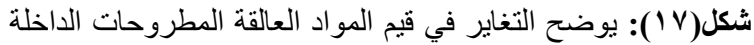

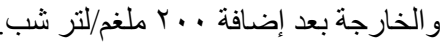

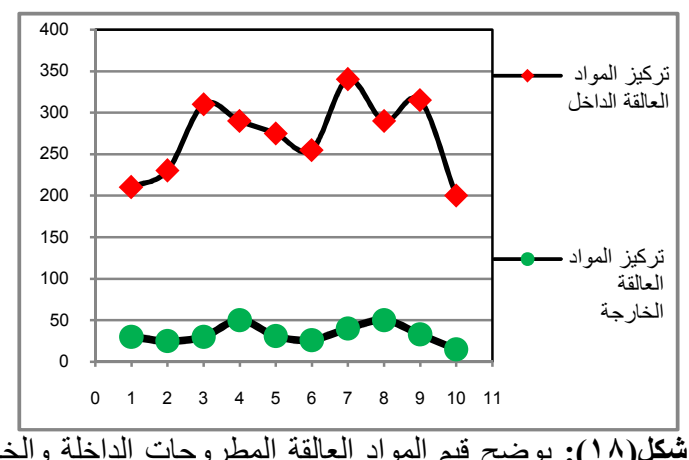

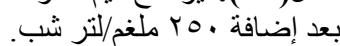

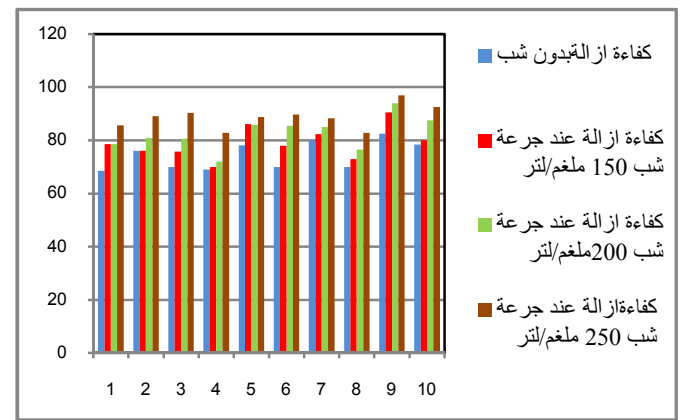

شكل(9 1): يوضح النسب المئوية لإزالة المواد العالقة في

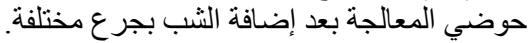

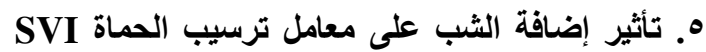
عند الجرع المختلفة

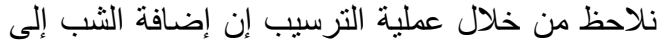

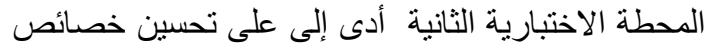

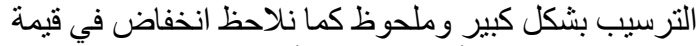

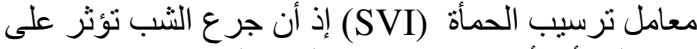

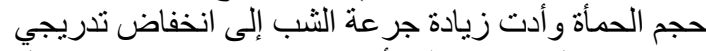

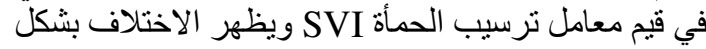

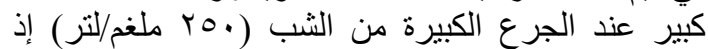

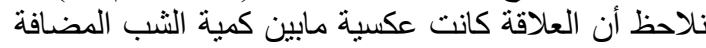

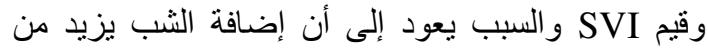
المساحة السطحية للجزيئات العالقة وبالتالي يحسن إلى قابلية

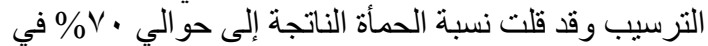

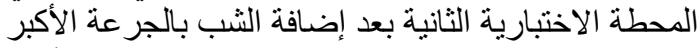

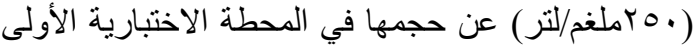
و هذا يتفق على ما جاء بـ (Daigger et al.,1985)

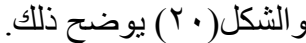

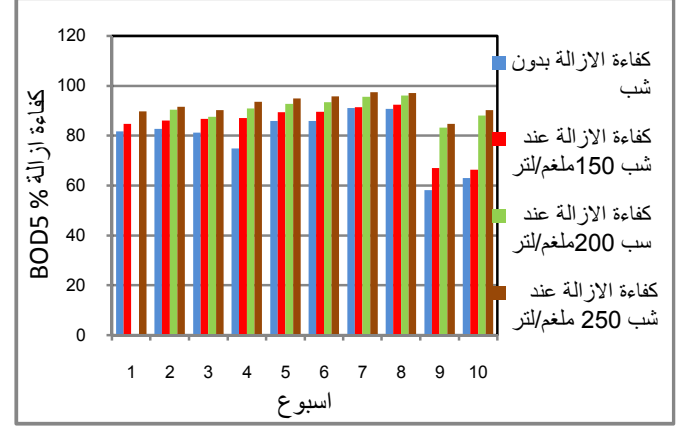

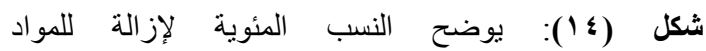

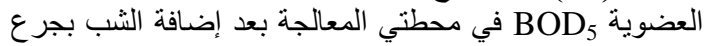
مختلفة الى المحطة الثنانية.

ك .تأثير إضافة الثب على تراكيز S.S S الخارجة عند

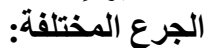

نلاحظ وجود فرق في قيمة المو اد العالقة الخارجة من القنة

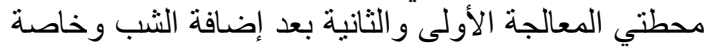

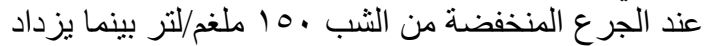

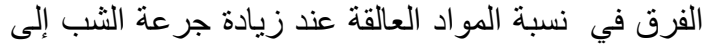
•

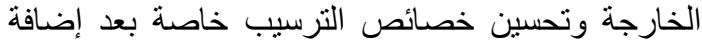

الجرع الكبيرة من الشب وهذا يتفق مع ما توصل إليه (Duker et al.,2007), (Daigger et al.,1985) أن إضافة الثب تحسن عملية الترسيب من خلال تكوين

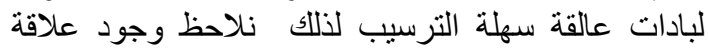

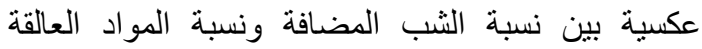
الخارجة. و الاشكال (0 1 ـ 9 (1) ) توضح ذلك.

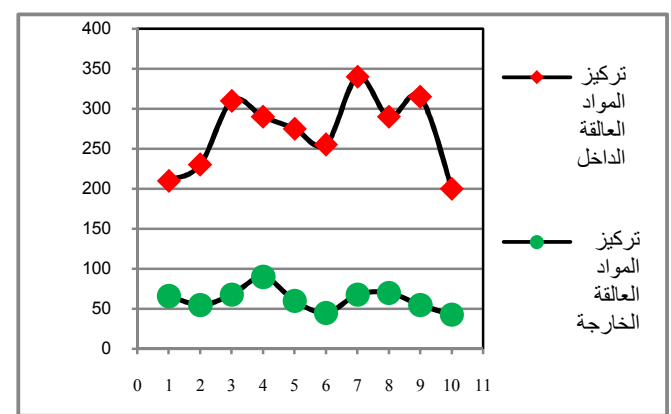

شكل(0) 10): يوضح التغاير في قيم المواد العالقة للمطروحات الداخلة و الخارجة من المحطة الاختبارية الأولى. فئ.

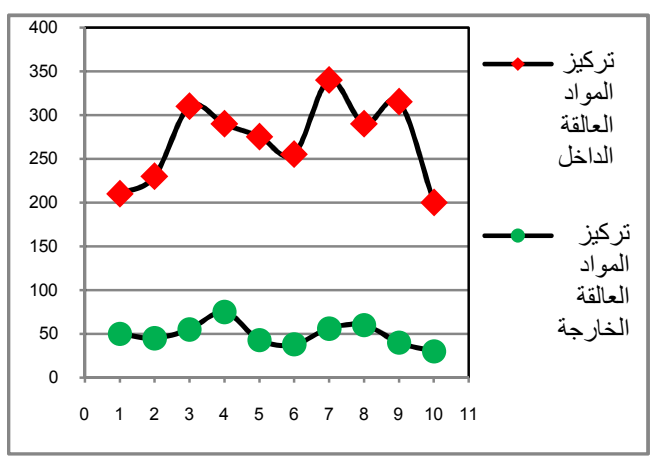

شكل(7 1): يوضح التغاير في قيم المواد العالقة للمطروحات

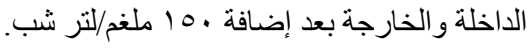


Barth, E.F. \& Etting, M.B. "Mineral controlled phosphorus removal in the Activated sludge processes "Journals water pollution control federation “39,8,1362(1976).

Gersbery, R.M., lyon, S.R., Brenner, (1988). "performance of Clay-Alum Flocculation (CCBA) process for Virus Removal From Municipal wastewater ". Journal of Water Research, Vol. 22, No. 11, P. 1449

Georgantas, D., Grigoropoulou, H.P., (2006). Phosphorus and organic matter removal from synthetic waster using alum and aluminum hydroxide. Global NEST J., 8 (2), 121-130.

AWWA; WPCF (1998). "Standard methods for the examination of water and wastewater", $20^{\text {th }}$ ed., Am. Public Healthy Assoc. Washington, D.C., USA

Plaza, E., Levlin, E., Hultman, B., (1997).

Phosphorus removal from wastewatera literature review. Division of Water Resources Engineering, Department of Civil and Environmental Engineering, Royal Institute of Technology, Stockholm

De Hass, D.W., Wentzel, M.C., Ekama, G.A. (2000). The use of simultaneous chemical precipitation in modified activated sludge systems exhibiting biological excess phosphate removal Part 1: Literature review. Water S.A., 26(4), 439-452.

Lujubinko, L., Julianna, G., Mirjana, D., Tatjana, K. (2004). Optimization of $\mathrm{pH}$ value and aluminium sulphate quantityin the chemical treatment of molasses. Eur. Food Res. Tech, 220, 70-73.

Juker, P. \&Hatch, M. "Impact of chemical addition in water/wastewater treatment on TDS concentration and sludge generation, JWW, (27), No (8), (2007).

Daigger, G.T. and Roper, R.E., Jr. (1985). The Relationship between SVI and Activated Sludge Settling Characteristics, J. Water Pollut. Control Fed., 57, 859.

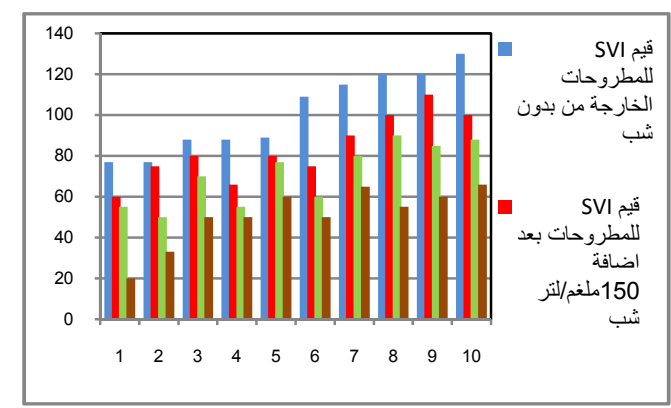

شكل (·r): يوضح قيم SVI في حوضي المعالجة بعد اضافة الثب بجر ع مختلفة. الاستنتاجات:

1- أظهرت الدراسة أن إضافة الثب إلى المحطة

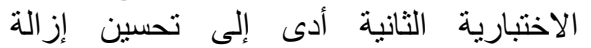

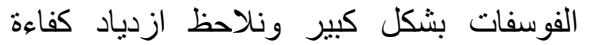

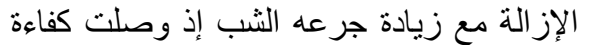

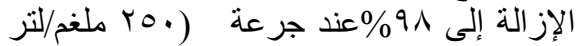

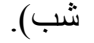

r- زيادة نسبة المو اد العالقة الخارجة من المحطة

الاختبارية الثانية بعد إضافة الثباد الثبة وتحسين الثانية

خصائص الترسيب بعد إضافة الثب الثب إلا انه

بمكن القضاء على هذه المشكلة بزيادة وقت الته

التعويق الهيدروليكي في حوض التهن التهوية.

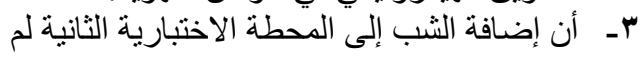

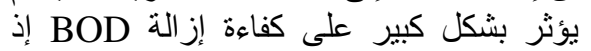

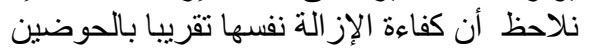

مما يدل على أن جميع المواد العادية العالقة الخارجة من الحوض كانت لا عضوية.

؛ - - إضافة الثب إلى المطروحات ألى أدت إلى تحسين

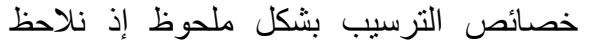

انخفاض في قيمة SVI مع زيادة نسبة الثب

المضناف .

Metcalf \& Eddy, Ins, "Wastewater engineering treatment disposal McGraw-Hill, Inc, New York. and reuse", Fourth Edition. (2003). pp477521.

David, R. \& EPA region "Advanced wastewater treatment to Achieve low concentration of phosphorus "Environmental protection agency, United state, Alaska, April (2007).

Wang, Y., Han, T., Xu Bao, G., Tan, Z. (2005). Optimization of phosphorus removal from secondary effluent using simplex method in Tianjin, China. J. Hazard. Mater, 21, 183-186.

Mahmut, O., Ayhan, S., Effect of tannins on phosphate removal using alum. Turkish J. Eng. Environ. Sci., 27, 227236. (2003). 


\title{
Effect of Alum Addition on the Biological Removal Efficiency and phosphates Removal
}

\author{
Zena Fakhri, Hanan Haqe \\ Env.research center \\ Env Eng.Dept
}

\begin{abstract}
The study aims to focus on the problem of excess nutrients in the discharges of treatment depends on the use of alum, or so called aluminum sulfate water. $\left(\mathrm{Al}_{2} 18(\mathrm{SO} 4)_{3}\right.$.), one of the materials used in the coagulation in the removal of phosphates and from the wastewater and by adding alum directly to the aeration tank through biological treatment process-based on continuous flow Activated sludge with different dose $(150,200,250 \mathrm{mg} / \mathrm{L})$. A comparisons Has been drawn between two basin to find the efficiency of removal the first without adding alum and the second was adding Alum at different Dose. The results showed the efficiency of alum in improving the removal of phosphate significantly since arrived removal efficiency to $98 \%$ at doses of $250 \mathrm{mg} / \mathrm{L}$ and improving the properties of sedimentation and its not affected widely on the efficiency removal of the BOD. As the $\mathrm{pH}$ values decreased gradually but did not affect the efficiency of removal in the basin of the treatment. Changing has been happened on the properties of the sludge in the basin as sludge change color to White and Milk after adding alum. The study proved the possibility of using alum in the treatment to improve the properties of discharges.
\end{abstract}

Key words: aluminum, nutrients, Activated sludge, phosphates biological treatment 